\title{
A New Method of Active Power Measurement Based on Power Load Wiener-G Functionals Models
}

\author{
Zhang Xiaobing, Yang Mengchen and Cao Wei \\ College of Measure-control Technology \& Communication Engineering \\ Harbin University of Science and Technology, Harbin, China \\ E-mail:Zhxiaobing@163.com
}

\begin{abstract}
For the problem of electrical energy measurement could not be united under the condition of non-stationary distortion signals, in the paper, the functionals series was used to modeling the signals. Firstly, the Wiener kernel of nonlinear load was acquired; the output signal of nonlinear load was expressed using functionals series under the condition of sinusoidal excitation; and then, we used the wavelet transform to processed and acquired component of fundamental and distortion; and though the acquisition component of voltage and current, we analyzed the power flow of nonlinear load, and according the result of power flow, we proposed a new method of active power measurement. The simulation result shows that the result of theoretical analysis is the same as the new method, and the new method of electric energy measurement based on the functional series can achieve the reasonable electrical energy measurement.
\end{abstract}

Keywords: Electrical energy measurement; Power flow; Functional series; Wavelet transform

\section{Introduction}

With the rapid increase of nonlinear load in power gird, the signal of power grid keeps deteriorating [1-3]. In the environment of the distortion power system, there's a complex energy exchange between nonlinear load and power grid, which was difficult for us to accurately measure the electrical energy of nonlinear load.

At present, there's some problems about the method of measuring the electrical energy under the condition of distortion. For example, under the condition of harmonic, according to the traditional electrical energy measurement method, the users of linear load not only need to pay the costs of fundamental power, but also to burden the costs that was forced to absorb harmonics energy. As to the nonlinear load, on the contrary, but the users burden little the costs that harmonic electricity energy pumped into the power system [45]. Obviously, it is extremely negative impact on improving the power quality. A reasonable method is to use the technical scheme of fundamental wave watt-hour meter, which has been successfully applied to the non-linear loads of using electric energy measurement, and achieved some results [6-7]. However, under the condition of harmonic, the electric energy measurement theory, method and instrument can't solve the universally accurate measurement of electricity in the condition of distortion signal. Especially the Non-stationary voltage and current signal of the nonlinear load such as impact loads, it can't be described by the mathematical model of harmonic signals [8].

In this paper, we established the general mathematical model of power grid signal using the Wiener-G functional series, and on the basis of that, we analyzed the power flow of nonlinear load and proposed the new method of energy metering. The simulation result shows that the new method of electrical measurement that proposed according to the analysis of power flow theoretically solved the problem of united energy measurement. 


\section{The Mathematical Models of power Grid Signals and Power under the Conditions of Distortion Signals}

\subsection{The Simplified Model of Power Grid}

As shown in figure 1, the simplified model of power grid signals under the conditionals of distortion signals. The power supply voltage of power grid is $u(t)$ and the current is $i(t), Z_{l}$ is the impedance of circuit, $Z$ is the impedance of nonlinear load, $a$ is the energy measurement nodes of load.

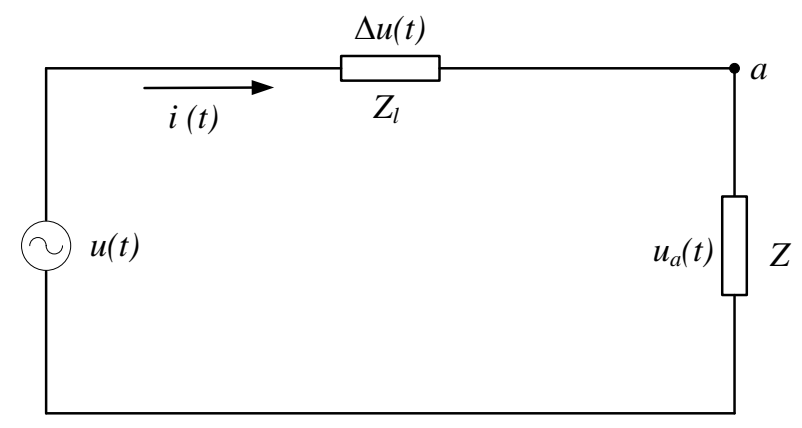

Figure 1. The Electrical Network Simplified Model

The voltage, current and its decomposition of nonlinear load are defined as follows

$$
\begin{gathered}
u_{a}(t)=u_{I}(t)+u_{s}(t) \\
i(t)=i_{I}(t)+i_{s}(t)
\end{gathered}
$$

Where $u_{I}(t)$ and $i_{I}(t)$ are the fundamental component of power signal, $u_{s}(t)$ and $i_{s}(t)$ are the distortion component of power signals.

The voltage drop of circuit impedance can be express as follow

$$
\Delta u(t)=\Delta u_{I}(t)+\Delta u_{s}(t)
$$

Where the $\Delta u_{I}(t)$ and $\Delta u_{s}(t)$ are the fundamental and distortion component of the voltage drop of circuit impedance respectively.

In the simplified model of power grid, $u_{s}(t), i_{s}(t)$ and $\Delta u_{s}(t)$ are random signals, they include not only harmonic component, also include other forms of distortion such as DC, inter-harmonic components.

\subsection{The Mathematics Model of Power under the Conditions of Distortion}

By the theory of power, we know the instantaneous power of $a$ point

$$
p_{a}(t)=u_{a}(t) i(t)
$$

Will (1) and (2) into (3)

$$
\begin{aligned}
p_{a}(t)= & {\left[u_{I}(t)+u_{s}(t)\right]\left[i_{I}(t)+i_{s}(t)\right] } \\
& =u_{I}(t) i_{I}(t)+u_{I}(t) i_{s}(t)+u_{s}(t) i_{I}(t)+u_{s}(t) i_{s}(t) \\
& =p_{I}(t)+p_{I s}(t)+p_{s I}(t)+p_{s}(t)
\end{aligned}
$$

The average power of a point is 


$$
\begin{aligned}
P_{a} & =\frac{1}{T} \int_{0}^{T} p_{a}(t) d t \\
& =\frac{1}{T} \int_{0}^{T}\left[p_{I}(t)+p_{I s}(t)+p_{s I}(t)+p_{s}(t)\right] d t \\
& =\frac{1}{T} \int_{0}^{T} p_{I}(t) d t+\frac{1}{T} \int_{0}^{T} p_{I s}(t) d t+\frac{1}{T} \int_{0}^{T} p_{s I}(t) d t+\frac{1}{T} \int_{0}^{T} p_{s}(t) d t \\
& =P_{I}+P_{I s}+P_{s I}+P_{s}
\end{aligned}
$$

Where $P_{I}$ is the fundamental component of power, $P_{I s}$ is produced by the fundamental component of voltage and distortion component of current, $P_{s I}$ is produced by distortion component of voltage and fundamental component of current and $P_{s}$ is produced by distortion component of voltage and distortion component of current(here, we called distortion power).

\section{Modeling the Power Grid Signal under the Condition of Distortion Signal and Wavelet Decomposition Algorithm}

The previous section shows, it is important to know the voltage and current model of nonlinear load, if we want to measure the consumptive electrical energy of nonlinear load.

1) The mathematical model was unified description using functional series.

2) The component of fundamental and distortion were acquired using the wavelet transform.

\subsection{Wiener Kernel Acquisition of Non-linear Systems}

According to the reference [9], and the nature of functional series, we have the Wiener kernel of nonlinear load:

$$
k_{n}\left(\tau_{1}, \cdots, \tau_{n}\right)=\frac{1}{n ! A^{n}} \mathrm{E}\left[i_{n-1}(t) \prod_{i=1}^{n} u\left(t-\tau_{i}\right)\right]
$$

Where $i_{n-1}=i(t)-\sum_{m=0}^{n-1} G_{m}(t), n>0$. $A$ is the power spectral density of input Gaussian White noise; $G_{\mathrm{m}}(t)$ is the items of Wiener series; $i(t)$ is the output of non-linear system, $u(t)$ is the input of non-linear system.

$$
\begin{aligned}
G_{m}\left[k_{m} ; u(t)\right]= & \sum_{i=0}^{[m / 2]} \frac{(-1)^{i} m ! A^{i}}{2^{i}(m-2 i) ! i !} \times \int_{-\infty}^{\infty} \cdots \int_{-\infty}^{\infty} k_{m}\left(\sigma_{1}, \ldots, \sigma_{m-2 i}, \tau_{1} \cdots, \tau_{i}, \tau_{i}\right) d \tau_{1} \ldots d \tau_{i} \times \\
& \prod_{r=1}^{m-2 i} u\left(t-\sigma_{r}\right) d \sigma_{1} \cdots d \sigma_{m-2 i}
\end{aligned}
$$

And then the Wiener kernel could be acquired.

\subsection{Wiener-G Functional Series Expression under the Condition of non-Gaussian White Noise Input}

If the input $u(t)$ of non-linear power system is non-Gaussian White Noise, the output $i(t)$ of system can be described as a mutually orthogonal of series, that is to say

$$
i(t)=\sum_{m=0}^{\infty} L_{m}\left[h_{m}\left(\tau_{1}, \cdots, \tau_{m}\right), u(t)\right]
$$

Where, $L_{\mathrm{m}}$ is the items of Wiener series, it is a function of input $u(t)$ and Wiener kernel $h_{\mathrm{m}}\left(\tau_{1}, \ldots, \tau_{\mathrm{m}}\right)$. The items are: 


$$
\begin{gathered}
L_{0}\left[h_{0} ; u(t)\right]=h_{0} \\
L_{1}\left[h_{1} ; u(t)\right]=\int_{-\infty}^{\infty} \int_{-\infty}^{\infty} h_{1}\left(\sigma_{1}\right) k_{1}\left(\tau_{1}-\sigma_{1}\right) u\left(t-\tau_{1}\right) d \sigma_{1} d \tau_{1} \\
L_{2}\left[h_{2} ; u(t)\right]=\int_{-\infty}^{\infty} \int_{-\infty}^{\infty} \int_{-\infty}^{\infty} \int_{-\infty}^{\infty} h_{2}\left(\sigma_{1}, \sigma_{2}\right) k_{1}\left(\tau_{1}-\sigma_{1}\right) k_{2}\left(\tau_{2}-\sigma_{2}\right) u\left(t-\tau_{1}\right) u\left(t-\tau_{2}\right) d \sigma_{1} d \sigma_{2} d \tau_{1} d \tau_{2} \\
-2 \pi \int_{-\infty}^{\infty} h\left(\sigma_{1}-\sigma_{2}\right) d \sigma_{2} \\
\ldots
\end{gathered}
$$

Where $k_{1}(t)=\frac{1}{2 \pi} \int_{-\infty}^{\infty} \frac{1}{\Phi_{u u}^{+}(\omega)} e^{j \omega t} d \omega$, and $\Phi_{\text {uu }}{ }^{+}(\omega)=C$ is the power spectral density of input signal $u(t), \Phi_{u u}^{+}(\omega)=\int_{-\infty}^{\infty} u(t) \bullet e^{-j \omega t} d t$, If the input signal of (4) is Gaussian White Noise, then the $\Phi_{\mathrm{uu}}{ }^{+}(\omega)=C$, where $C$ is constant, the output electric parameters of power load can be described as (4) no matter what the input is.

The $u_{a}(t)$ could be acquired by $i(t)$ :

$$
u_{a}(t)=u(t)-R_{l} i(t)
$$

Where $u(t)$ is power voltage, $R_{l}$ is impedance of line

\subsection{Wavelet Decompose and Reconstruction}

Based on the functionals series model voltage and current signal, using the wavelet algorithm decompose and reconstruct the voltage and current, we can find the component of fundamental and the component of distortion under the conditions of distortion signals[10].

From the MRA(Multi-resolution Analysis) theory, $u(t)$ and $i(t)$ can be broken down into different frequency components

$$
\begin{aligned}
& u(t)=\sum_{k} c_{0}(k) \varphi(t-k)+\sum_{k} \sum_{j=0}^{J-1} d(k) 2^{j / 2} \psi\left(2^{j} t-k\right) \\
& i(t)=\sum_{k} c_{0}^{\prime}(k) \varphi(t-k)+\sum_{k} \sum_{j=0}^{J-1} d_{j}^{\prime}(k) 2^{j / 2} \psi\left(2^{j} t-k\right)
\end{aligned}
$$

Where $J$ is the level of decomposition, $c_{j}(k)$ and $c_{j}^{\prime}(k)$ is the scale coefficient, $d_{j}(k)$ and $d_{j}^{\prime}(k)$ is the wavelet coefficient. $\varphi(t)$ is the scale function, $\psi(t)$ is the wavelet function. The voltage and current could be decompose to different frequency band from initial-scale coefficients layer by layer.

The different frequency band wavelet coefficients of voltage and current could be expressed matrix form:

$$
\left.\begin{array}{l}
C_{\text {signal }}=\left[c_{0, k}, d_{0, k}, \cdots, d_{J-1, k}\right] \\
C_{\text {signal }}^{\prime}=\left[c_{0, k}^{\prime}, d_{0, k}^{\prime}, \cdots, d_{J-1, k}^{\prime}\right]
\end{array}\right\}
$$

Similarly, the wavelet coefficients matrix $\mathbf{C}_{\text {basis }}, \mathbf{C}_{\text {basic }}^{\prime}, \mathbf{C}_{\text {rand }}$ and $\mathbf{C}_{\text {rand }}^{\prime}$ of $u_{I}(t)$, $i_{I}(t), u_{s}(t)$ and $i_{s}(t)$, respectively. 
According to reconstruction equation as follow, the distortion voltage and current were acquired

$$
\left.\begin{array}{l}
u_{s}^{\prime}(t)=\sum_{k} c_{0}^{u} \varphi(t-k)+\sum_{k} \sum_{j=0}^{J-1} d_{j}^{u}(k) 2^{j / 2} \psi\left(2^{j} t-k\right) \\
i_{s}^{\prime}(t)=\sum_{k} c_{0}^{i} \varphi(t-k)+\sum_{k} \sum_{j=0}^{J-1} d_{j}^{i}(k) 2^{j / 2} \psi\left(2^{j} t-k\right)
\end{array}\right\}
$$

Similarly, the component of fundamental voltage and current could be reconstruction.

By now, the signal of any nonlinear load could be expressed to functional series, if the Wiener kernel could be obtained.

\section{Simulation Test of Load Power Flow and Error Analysis}

In order to verify the functionals series model, according to the functionals series model of nonlinear load and the decomposition algorithm of signal, in this section, we have a power flow simulation experiment and error analysis about three type nonlinear load and its composite nonlinear load.

\subsection{Power Flow Analysis of Electrical arc Furnace}

We take an electric arc furnace of steel factory for an example. According to the characteristic of electrical arc furnace, the theoretical model was acquired by simulink modeling, the simulation model was acquired by functionals series modeling. $f_{\mathrm{s}}=3200 \mathrm{~Hz}$ is sampling frequency of simulation, $\mathrm{n}=256$ is sampling points, and the signal is decomposed into 4 levels, the components of fundamental and distortion were acquired.

The figure 2 is wavelet decomposition of voltage and current under the condition of distortion signal. The table 1 is simulation result of voltage and current under the condition of distortion signal.

Table 1. The Simulation Results

\begin{tabular}{cccccc}
\hline & $P_{a}$ & $P_{I}$ & $P_{I s}$ & $P_{s I}$ & $P_{s}$ \\
$\begin{array}{c}\text { Theoretical } \\
\text { value(W) }\end{array}$ & 153.815 & 158.370 & 21.654 & -14.514 & -11.695 \\
$\begin{array}{c}\text { Measured } \\
\text { value(W) }\end{array}$ & 145.293 & 147.594 & 19.844 & -13.078 & -11.067 \\
Error & $-5.5 \%$ & $-6.8 \%$ & $8.4 \%$ & $9.9 \%$ & $-0.5 \%$ \\
\hline
\end{tabular}

From the simulation result, we know

1) The power accuracy level is $10^{-2}$, the direction of fundamental and distortion power flow is same as theoretical situation, it shows that the functional series models could be apply to analysis power flow of the impact nonlinear load.

2) $P_{I s}$ and $P_{S I}$ are not zero, it shows the fundamental meter ignore these two item is neither reasonable nor accurate.

3) In this simulation instance, distortion power accounted for $7.6 \%$ of total power, but with the increase of the load, the effects are not negligible. 


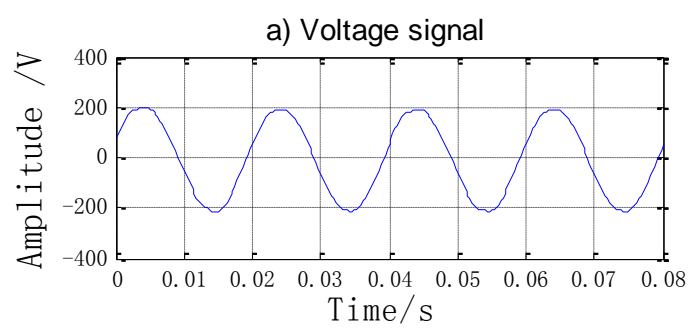

b) The fundamental voltage of reconstruction

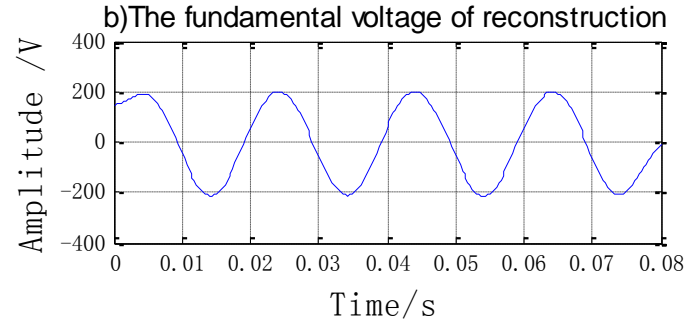

c) The distortion voltage of reconstruction

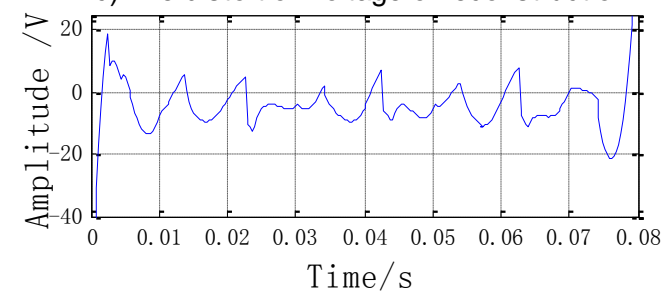

d) Current signal

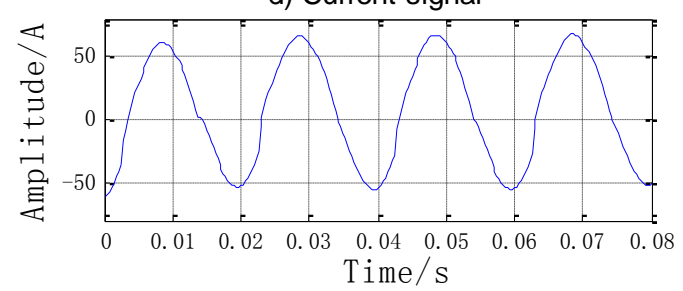

e)The fundamental current of reconstruction
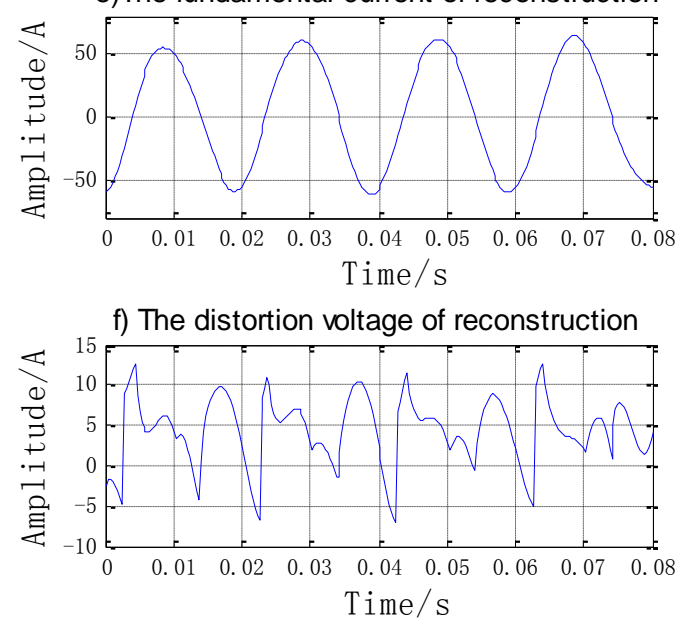

Figure 2. Current and Voltage of Semiconductor Rectifier and its Decompose

\subsection{Power Flow Analysis of Composite System}

The theoretical model of composite system contain the semiconductor rectifier device of the conduction angle $\alpha$ is $\pi / 4$, SS1 electric locomotive-type load and electrical arc furnace, of which the semiconductor rectifier device and the SS1electric locomotive-type load had been analyzed. The frequency $f_{\mathrm{s}}=3200 \mathrm{~Hz}$ is sampling frequency of simulation, $\mathrm{n}$ $=256$ is sampling points, and the signal is decomposed into 4 levels, the components of fundamental and distortion were acquired.

The figure 3 is wavelet decomposition of voltage and current under the condition of distortion signal. The table 2 is simulation result of voltage and current under the condition of distortion signal.

Table 2. The Simulation Results

\begin{tabular}{c|c|c|c|c|c}
\hline & $P_{a}$ & $P_{I}$ & $P_{I s}$ & $P_{s I}$ & $P_{s}$ \\
\hline $\begin{array}{c}\text { Theoretical } \\
\text { value(W) }\end{array}$ & 880.4649 & 926.8676 & 27.1569 & -37.1176 & -36.4421 \\
\hline $\begin{array}{c}\text { Measured } \\
\text { value(W) }\end{array}$ & 870.8557 & 913.5854 & 25.3569 & -33.5980 & -34.4886 \\
\hline Error & $-1.1 \%$ & $-1.4 \%$ & $-6.6 \%$ & $9.5 \%$ & $-5.4 \%$ \\
\hline
\end{tabular}

From the simulation result, we know

1) The composite system power accuracy level is $10^{-2}$, the direction of fundamental and distortion power flow is same as theoretical situation, it shows that the functional 
series models could be apply to analysis power flow of composite system with many nonlinear load.

2) The error level of composite system is same as single system, it shows that the unify power flow analysis is feasible for composite system.
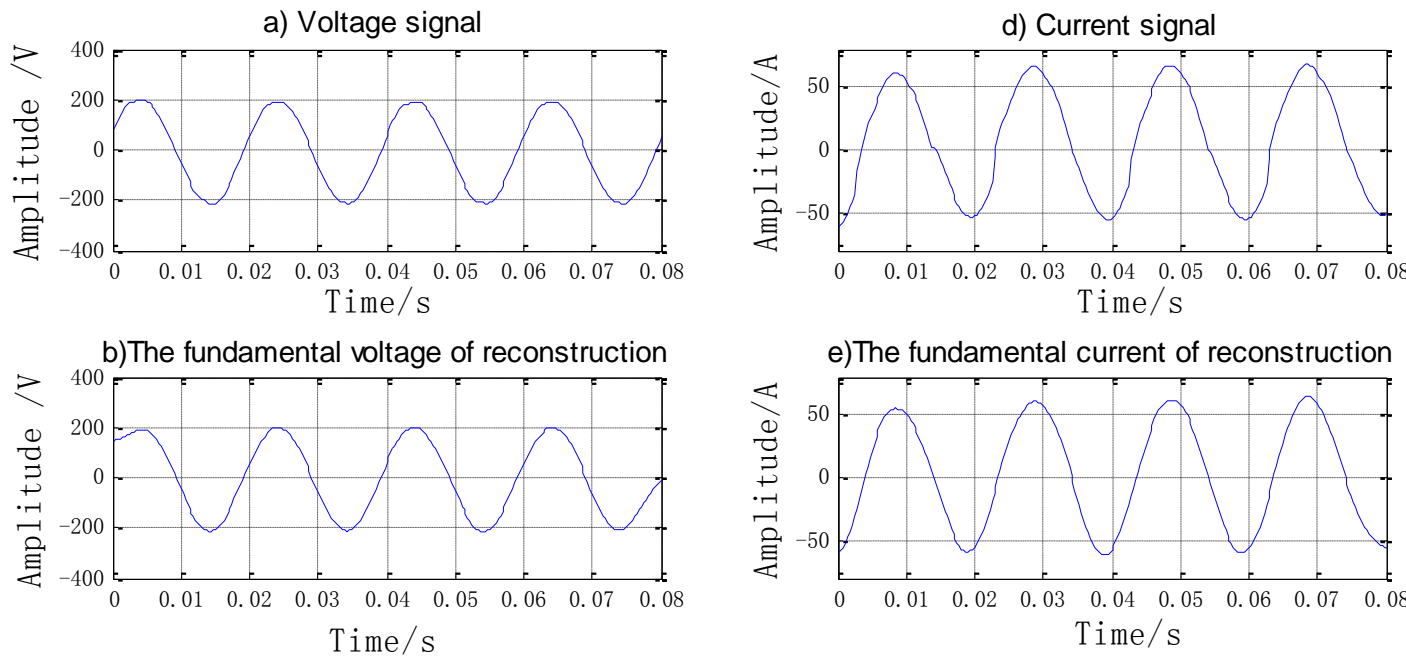

e)The fundamental current of reconstruction

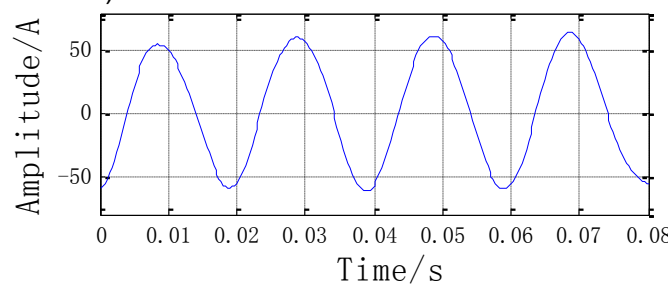

c) The distortion voltage of reconstruction
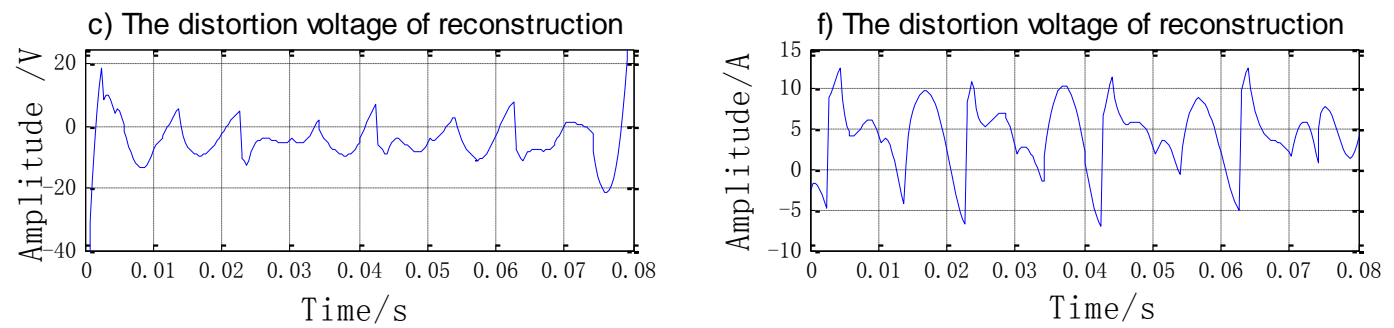

Figure 3. Current and Voltage of Composite Load and its Decompose

\section{A New Method of Electrical Energy Measurement under the Conditions of Distortion}

From the result of power flow analysis and simulation, we can available that:

What the power was produced by fundamental components of voltage and current is $P_{I}>0$, it indicate that the nonlinear load absorption fundamental power from power grid, so this part of electrical energy should be include into total energy of load consumption.

What the power was produced by fundamental component of voltage and distortion component of current that is $P_{I s}>0$, it indicate that the nonlinear load absorption distortion power from power grid, so this part of electrical energy should be measure.

What the power was produced by distortion component of voltage and fundamental component of current is $P_{s I}<0$, it indicate that this component power is not be consumption, but as the distortion power back to the grid. However, the form of current back to the power grid is fundamental, and don't bring pollution to the power grid, so it should be measured.

What the power was produced by distortion component of voltage and current is $P_{s}>0$, it indicate that this part of power not been consumption, but as the distortion power back to the power grid. However, the form of current back to the power grid is distortion, and bring pollution to the power grid, therefore not be measured. 
In summary, we proposed a universal method of electrical measurement for the nonlinear load

$$
\begin{gathered}
P=P_{I}+P_{I s}+P_{s I} \\
P=P_{a}-P_{S}
\end{gathered}
$$

Where, $P_{a}$ is the measure power of measurement node $a, P_{s}$ is the distortion part of $P_{a}$.

When the $u_{s}(t)$ and $i_{s}(t)$ are the higher-order harmonics, type become on

$$
P=P_{I}+P_{I S}+P_{s I}=P_{I}
$$

According to the type, we manufacture the meter of electrical energy measurement degenerate as a fundamental watt-hour meter, so it also applies to the harmonic user.

\section{Conclusion}

In this paper, the functionals series model of nonlinear load was regarded as basis to analyse the power flow of nonlinear load, and according to the result of power flow, we proposed a new method of electrical energy measurement, and got the conclusion as following:

1) It is different between simulation and theoretical components of power value what the composite system contains the semiconductor rectifier device, electric locomotive-type load and electrical arc furnace, and the error level is $10^{-2}$. The main reasons of simulation error are the speed and memory storage which limit the usage of the functional series model proceed power flow analysis, on one hand just a little sample data can be used to computations, one the other hand, only a few items of functional series can be used to approximate the practical system.

2) The simulation result of power flow direction of composite system contain the semiconductor rectifier device, electric locomotive-type load and electrical arc furnace that are consistent with theoretical results, and at the same time, the results are consistent with theoretical analysis results of article[8], it shows that the functional series models could be apply to analysis power flow of composite system with many nonlinear load, and also the new method could be used to measure electrical energy.

3) In order to further validate the universality of the new method of electrical energy measurement, the authors are developing the experiment instrument of electrical energy measurement

\section{Acknowledgments}

This work was financially supported by the National Natural Science Foundation (51277043).

\section{References}

[1] Y. Xing and B. Li, "The impact of nonlinear load on the voltage and current of the grid [J]", Power System, vol. 26, no. 8, (2007), pp. 25-28.

[2] Y. Haiwen, Q. Ye and Z. Cheng, "Research on harmonic current distribution of Beijing's rail traffic power supply system [C]", 9th International Conference on Electronic Measurement \& Instruments, Beijing, China: The Chinese Institute of Electronics, (2009), pp. 235-238.

[3] J. Zhao, F. Wen and A. Yang, "Impacts of electric vehicles on power systems as well as the associated dispatching and control problem [J]", Automation of Electric Power Systems, vol. 35 , no. 14, (2011), pp. 2-10, 29. 
[4] J. Yao, T. Zhang and R. Lin, "Impacts of negative sequence current harmonics in traction power supply system for electrified railway on power system and compensation measures [J]", Power System Technology, vol. 32, no. 9, (2008), pp. 61-64, 88.

[5] W. Yan, P. Jiang and F. Li, "Effects of harmonics on electric energy metering [J]", East China Electric Power, vol. 34, no. 2, (2006), pp. 40-42.

[6] W. Jin and G. Liu, "Research on the fundamental electric energy meter based on active filter and its application [J]", Journal of Changsha University of Electric Power (Natural Science), vol. 18 , no. 4, (2003), pp. 41-44.

[7] G. Yunpeng, Z. Teng and H. Wen, "Harmonic analysis based on Kaiser window interpolation FFT and its application [J]", Proceedings of the CSEE, vol. 30, no. 4, (2010), pp. 43-48.

[8] X. Zhang, "Electrical network power flow analysis and research on new measuring methods of electric energy on the condition of distorted signals [D]", Harbin: Harbin University of Science and Technology, (2007), pp. 5-31.

[9] X. Zhang, Y. Li and G. Fang, "Modeling for Grid Signals Under the condition of nonstationary distortion signals [J]", Proceedings of the CSEE, vol. 34, no. 16, (2014), pp. 27212728.

[10] X. Zhang, J. Cui and Y. Liang, "The Application of Wavelet Transform in Power Quality Disturbance Detection and location [J]", Journal Harbin UNIV. SCI \& TECH, vol. 11, no. 06, (2006), pp. 118-121. 
International Journal of Signal Processing, Image Processing and Pattern Recognition Vol.8, No.10 (2015) 